Bull. Soc. math. France

131 (2), 2003, p. 229-257

\title{
SUR LA DYNAMIQUE ARITHMÉTIQUE DES AUTOMORPHISMES DE L'ESPACE AFFINE
}

\author{
PAR SANDRA MARCELLO
}

\begin{abstract}
RÉSumÉ. - Nous étudions les propriétés arithmétiques des itérés de certains automorphismes polynomiaux affines. Nous traitons des questions concernant les points périodiques et non-périodiques, en particulier nous comptons les points rationnels dans les orbites des points non-périodiques. Nous traitons le cas des automorphismes réguliers et triangulaires. Nous achevons de répondre aux questions en dimension 2 et montrons que la situation est nettement plus compliquée en dimension supérieure.

ABSTRACT (On the arithmetic dynamic of automorphisms of the affine space)

We study arithmetic properties of the iterates of some affine automorphisms. We study questions concerning periodic and non periodic points, in particular counting rational points in orbits of non-periodic points. We study the case of the regular and the triangular automorphisms. We finish to answer to the questions in dimension 2 and show that the situation is much more complicated in greater dimension.
\end{abstract}

\section{Table des matières}

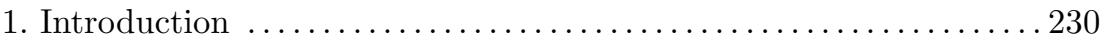

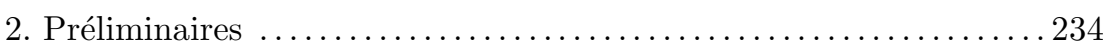

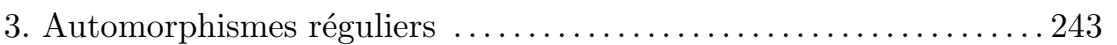

Texte reçu le 25 octobre 2001, révisé le 10 juin 2002, accepté le 9 septembre 2002

SANDra Marcello, Adresse permanente : Théorie des Nombres, Institut de Mathématiques de Jussieu, 175, rue du Chevaleret, 75013 Paris (France). Adresse actuelle : MPIM, P.O. Box : 7280, D-53072 Bonn (Deutschland) • E-mail :marcello@math.jussieu.fr et marcello@mpim-bonn.mpg.de

Classification mathématique par sujets (2000). — 11G50, 32M17, 37F10.

Mots clefs. - Hauteurs, applications polynomiales, degré dynamique, itérés, points périodiques. 


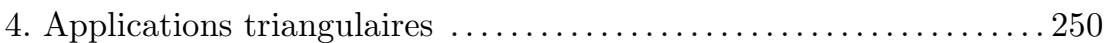

5. Automorphismes du plan affine ............................ 252

6. Différents exemples en dimension au moins $3 \ldots \ldots \ldots \ldots \ldots \ldots 253$

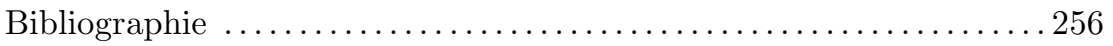

\section{Introduction}

Nous nous intéressons à des propriétés arithmétiques des itérés d'automorphismes (polynomiaux) affines; ceux-ci sont définis comme suit :

DÉFINITION 1.1. - On appelle morphisme polynomial affine ou morphisme affine, un morphisme de l'espace affine :

$$
\phi: \mathbb{A}^{r} \longrightarrow \mathbb{A}^{r}, \quad\left(x_{1}, \ldots, x_{r}\right) \longmapsto\left(F_{1}\left(x_{1}, \ldots, x_{r}\right), \ldots, F_{r}\left(x_{1}, \ldots, x_{r}\right)\right)
$$

avec $F_{i}$ application polynomiale pour $i=1, \ldots, r$.

Un morphisme possédant un inverse également polynomial est un automorphisme; l'ensemble des automorphismes polynomiaux de $\mathbb{A}^{r}$ est noté $\operatorname{Aut}\left(\mathbb{A}^{r}\right)$. vante

On appelle degré algébrique ou degré de $\phi$, l'entier défini de la manière sui-

$$
\operatorname{deg}(\phi)=d=\sup _{i}\left(d_{i}\right)
$$

avec $d_{i}$ degré total de $F_{i}$. Le degré dynamique (voir $\S 2.2$ ) est

$$
\delta(\phi):=\inf _{n}\left[\operatorname{deg}(\phi)^{n}\right]^{\frac{1}{n}}
$$

DÉfinition 1.2. - Soient $\phi, f$ deux automorphismes polynomiaux affines. L'application conjuguée de $\phi$ par $f$ est

$$
f^{-1} \circ \phi \circ f \text {. }
$$

Dans ce texte, sauf spécification contraire, le terme « conjugaison » désigne la conjugaison par un automorphisme polynomial affine. En dimension 2, il existe une classification des automorphismes du plan affine à « conjugaison » près [5]; les propriétés que nous étudions sont elles-mêmes essentiellement stables par « conjugaison».

Nous nous plaçons pour notre étude dans $\overline{\mathbb{Q}}$ où nous avons ainsi à notre disposition la notion de hauteur d'un point. Soient $k$ un corps de nombres, $M_{k}$ l'ensemble des places de $k$ et $v \in M_{k}$; nous définissons $n_{v}=\left[k_{v}: \mathbb{Q}_{v}\right]$ (nous avons adopté les notations de [6]).

DÉFINITION 1.3. - La hauteur absolue (multiplicative) sur $\mathbb{P}^{r}$ est la fonction

$$
H: \mathbb{P}^{r}(\overline{\mathbb{Q}}) \longrightarrow[1, \infty), \quad H(P)=H_{k}(P)^{1 /[k: \mathbb{Q}]},
$$

où $k$ est un corps de nombres, $P \in \mathbb{P}^{r}(k)$ et $H_{k}(P)=\prod_{v \in M_{k}} \max _{i}\left(\left|x_{i}\right|_{v}^{n_{v}}\right)$.

TOME $131-2003-\mathrm{N}^{\mathrm{O}} 2$ 
La hauteur absolue (logarithmique) sur $\mathbb{P}^{r}$ est

$$
h: \mathbb{P}^{r}(\overline{\mathbb{Q}}) \longrightarrow[0, \infty), \quad h(P)=\log H(P)=\frac{1}{[k: \mathbb{Q}]} \log H_{k}(P) .
$$

Soit $P \in \mathbb{A}^{r}(\overline{\mathbb{Q}})$; nous définissons comme suit sa hauteur :

$$
H(P)=H\left(x_{1}, \ldots, x_{r}, 1\right) .
$$

Une forme faible d'un théorème classique sur les hauteurs est : l'ensemble des points d'un espace projectif à coordonnées dans un corps de nombres est un ensemble fini (voir par exemple [6, B.2.3]). De plus, la notion de point périodique est une généralisation de la notion de point de torsion or, l'ensemble des points de torsion d'une variété abélienne définie sur un corps de nombres est un groupe fini (voir par exemple $[6, \mathrm{C}]$ ). La question suivante se pose donc de manière naturelle.

Question (Q1). — L'ensemble des points périodiques est-il de hauteur bornée?

Cependant si nous considérons le cas de l'identité, l'ensemble de ses points périodiques est clairement infini. Il est néanmoins possible de poser une question un peu plus faible, qui permettra notamment de donner un réponse globale pour tous les automorphismes du plan affine; la formulation de cette variante de la première question nécessite l'introduction de la notion suivante :

DÉFInItion 1.4. - Un point $P$ de l'espace affine est un point périodique isolé s'il existe $n \in \mathbb{N} \backslash\{0\}$ tel que $P$ est isolé (au sens de la topologie de Zariski) dans

$$
\left\{Q \in \mathbb{A}^{r} \text { tel que } \phi^{n}(Q)=Q\right\} .
$$

La variante de la question 1 se formule donc de la manière suivante :

Question (Q1'). - L'ensemble des points périodiques isolés sur un corps de nombres $k$ est-il fini?

Une réponse positive à la question $\left(\mathrm{Q} 1^{\prime}\right.$ ) est conjecturée (voir [16], [2]). Les questions (Q1) et $\left(\mathrm{Q} 1^{\prime}\right)$ parraissent également naturelles si l'on considère le résultat de Northcott [13] qui est que tout endomorphisme de variété lisse projective et de degré au moins 2 admet un nombre fini de points périodiques sur un corps de nombres.

Nous noterons $\operatorname{Per}(\phi, k)$, l'ensemble des points périodiques de $\phi$ à coordonnées dans le corps de nombres $k$.

Le second point étudié concerne les points non-périodiques, nous définissons, à l'aide de la hauteur, une fonction de comptage pour les orbites des points nonpériodiques et ce, de manière analogue aux problèmes de décompte de points rationnels des variétés (pour une synthèse, voir [14]). 
Question (Q2). - Soit $P$ un point non périodique. Nous définissons la taille de l'orbite d'un tel point comme la fonction

$$
N(B)=N(\phi, P, B)=\#\left\{\phi^{n}(P) \mid n \in \mathbb{Z}, h\left(\phi^{n}(P)\right) \leq B\right\} .
$$

Quel est le comportement asymptotique de cette fonction quand $B$ tend vers l'infini?

Plus précisément, on dira que

- $N(B)$ est comparable à $\log B($ noté $N(B) \gg \ll \log B)$ s'il existe $a_{1}, a_{2}, a_{3}, a_{4}$ dans $\mathbb{R}$ tels que

$$
a_{1} \log B+a_{2} \leq N(B) \leq a_{3} \log B+a_{4}
$$

- $N(B)$ est équivalent à $\log B$ (noté $N(B) \sim \log B$ ) s'il existe $a \in \mathbb{R}^{+}$tel que

$$
\lim _{B \rightarrow+\infty} \frac{N(B)}{\log B}=a .
$$

Dans le cas des automorphismes affines, J. Silverman et L. Denis ont déjà étudiés ces questions. J. Silverman [16] a répondu de manière affirmative à la question (Q1) et donné une réponse à la question (Q2) dans le cas des applications de Hénon quadratiques. Quant à lui, L. Denis [2] a répondu à ces différentes questions pour différentes applications; il a également répondu de manière affirmative à la question $\left(\mathrm{Q} 1^{\prime}\right)$ pour les automorphismes du plan affine.

Dans ce texte nous étudions différentes familles d'automorphismes qui outre leur intérêt propre — nous permettent de répondre à la question (Q2) pour les automorphismes du plan affine.

Soit $\phi: \mathbb{A}^{r} \rightarrow \mathbb{A}^{s}$ une application polynomiale définie comme suit :

$$
\phi\left(X_{1}, \ldots, X_{r}\right)=\left(F_{1}(X), \ldots, F_{s}(X)\right)
$$

avec $F_{i} \in \mathbb{C}\left[X_{1}, \ldots, X_{r}\right]$ pour $i=1, \ldots, s$ et $d:=\max _{i} \operatorname{deg}\left(F_{i}\right)$. L'application rationnelle $\widetilde{\phi}$ associée à $\phi$ est définie comme suit :

$$
\widetilde{\phi}: \mathbb{P}^{r} \longrightarrow \mathbb{P}^{s} \quad \text { avec } \tilde{\phi}\left(X_{1}, \ldots, X_{r}, X_{r+1}\right)=\left(\widetilde{F}_{1}(X), \ldots, \widetilde{F}_{s}(X), X_{r+1}^{d}\right),
$$

avec $\widetilde{F}_{i}$ polynôme homogène en $\left(X_{1}, \ldots, X_{r+1}\right)$ de degré $d$ associé à $F_{i}$ pour $i=1, \ldots, r$.

Une famille d'automorphismes affines se distingue naturellement en dynamique holomorphe [15] : il s'agit des automorphismes réguliers.

DÉfinition 1.5. - Soit $\phi$ un automorphisme de $\mathbb{A}^{r}, Z(\phi)$ désigne le lieu de non-définition de l'application rationnelle (définie sur $\mathbb{P}^{r}$ ) associée à $\phi$. L'automorphisme $\phi$ est dit régulier si

$$
\operatorname{deg}(\phi)>1 \text { et } Z(\phi) \cap Z\left(\phi^{-1}\right)=\varnothing .
$$

TOME $131-2003-\mathrm{N}^{\mathrm{O}} 2$ 
Soit $H$ l'hyperplan à l'infini; nous avons $Z(\phi) \subset H$.

En dimension 2, les applications de Hénon généralisées sont des automorphismes réguliers. Nous relions la dynamique arithmétique des automorphismes réguliers à leur géométrie.

ThÉORÈme A. - Soient $\phi$ un automorphisme régulier de $\mathbb{A}^{r}$ de degré $d$ et $\ell=\operatorname{dim} Z\left(\phi^{-1}\right)+1$. Alors :

- l'ensemble des points périodiques est un ensemble de hauteur bornée sur $\overline{\mathbb{Q}}$, $i l$ est a fortiori fini sur $k$;

- pour tout point non-périodique $P$ de $\mathbb{A}^{r}(\overline{\mathbb{Q}})$, nous avons

$$
N(\phi, P, B) \sim \frac{r}{\ell} \frac{\log B}{\log d} .
$$

Nous pouvons déduire de ce théorème un énoncé invariant par conjugaison.

Corollaire B. - Soit $\phi$ un automorphisme conjugué (par un automorphisme affine) à un automorphisme régulier. Alors :

- l'ensemble des points périodiques est un ensemble de hauteur bornée sur $\overline{\mathbb{Q}}$, il est a fortiori fini sur $k$;

- pour tout point non-périodique $P \in \mathbb{A}^{r}(\overline{\mathbb{Q}})$, nous avons

$$
N(\phi, P, B) \sim\left(\frac{1}{\log \delta(\phi)}+\frac{1}{\log \delta\left(\phi^{-1}\right)}\right) \log B .
$$

Nous obtenons également des résultats pour une autre famille d'automorphismes, les automorphismes triangulaires.

DÉfinition 1.6. - Un automorphisme $\phi$ de $\mathbb{A}^{r}$ est dit triangulaire s'il existe $F_{i} \in k\left[X_{i+1}, \ldots, X_{r}\right]$ pour $1 \leq i \leq r-1, F_{r} \in k$ et $a_{i} \in k^{*}$ pour $1 \leq i \leq r$ tel que

$$
\begin{array}{r}
\phi\left(X_{1}, \ldots, X_{r}\right)=\left(a_{1} X_{1}+F_{1}\left(X_{2}, \ldots, X_{r}\right), a_{2} X_{2}+F_{2}\left(X_{3}, \ldots, X_{r}\right)\right. \\
\left.\ldots, a_{r-1} X_{r-1}+F_{r-1}\left(X_{r}\right), a_{r} X_{r}+F_{r}\right) .
\end{array}
$$

ThÉORÈme C. - Soit $\phi$ une application triangulaire de $\mathbb{A}^{r}$. Soit $\mu^{\infty}$ l'ensemble des racines de l'unité.

- L'automorphisme $\phi$ admet un nombre fini de points de périodiques isolés dans $k$.

- Soit $P=\left(x_{1}, \ldots, x_{r}\right) \in \mathbb{A}^{r}(\overline{\mathbb{Q}})$ un point non-périodique. Alors :

$\triangleright$ s'il existe $i$ tel que $a_{i} \notin \mu^{\infty}$ et $x_{i} \neq 0$, on a $N(\phi, P, B) \gg \ll B$;

$\triangleright$ sinon $\log N(\phi, P, B) \gg \ll B$.

Ces résultats nous permettent, à l'aide de la classification à conjugaison près des automorphismes du plan affine, de retrouver un résultat de L. Denis [2] les concernant. Celui-ci [2] a en effet montré que tout automorphisme du plan affine admet un nombre fini de points périodiques isolés sur un corps de nombres $k$. 
Nous obtenons de plus, des renseignements sur les orbites des automorphismes du plan affine.

ThÉORÈme D. - Soit $\phi$ un automorphisme du plan affine.

- Si $\delta(\phi)=1$, alors $N(B) \gg \ll B$ ou $\log N(B) \gg \ll B$.

- Si $\delta(\phi) \neq 1$, alors $N(B) \sim 2 \log B / \log \delta(\phi)$.

Nous trouvons donc trois types d'orbites

$$
N(B) \sim c \log B, \quad N(B) \gg \ll B, \quad \log N(B) \gg \ll B
$$

en dimension 2. La question naturelle qui se pose est de savoir si ce sont les seuls comportements possibles. En dimension au moins 3, nous retrouvons également ces trois tailles d'orbites. Dans la dernière partie, nous déterminons les comportements de différentes applications qui montrent que la situation est nettement plus complexe en dimension au moins 3 .

Remerciements. - Ce texte est tiré de ma thèse, effectuée sous la direction de Marc Hindry, que je tiens à remercier pour ses conseils et sa disponibilité. Je remercie également Laurent Denis pour ses questions et ses remarques et enfin je remercie le referee pour sa lecture attentive de ce texte.

\section{Préliminaires}

Dans cette partie, nous définissons les notions, donnons des résultats que nous serons amenée à utiliser par la suite.

2.1. Notion de hauteur. - Nous avons rappelé dans l'introduction la notion de hauteur; nous énonçons ici certaines de ses propriétes que nous serons amenée à utiliser par la suite. Toutes les propriétés que nous énonçons ici se trouvent dans $[6, \mathrm{~B}]$.

La proposition suivante se déduit aisément de la définition de la hauteur.

Proposition 2.1. - Soit $\alpha_{1}, \ldots, \alpha_{n} \in k$ et $F \in k\left[X_{1}, \ldots, X_{r}\right]$. Nous avons :

1) $h\left(\alpha_{1}+\cdots+\alpha_{n}\right) \leq h\left(\alpha_{1}, \ldots, \alpha_{n}\right)+\log n$;

2) $h\left(F\left(\alpha_{1}, \ldots, \alpha_{r}\right)\right) \leq \operatorname{deg}(F) h\left(\alpha_{1}, \ldots, \alpha_{r}\right)+c(r, F)$;

3) $h\left(\alpha_{1}+\alpha_{2}\right) \leq h\left(\alpha_{1}\right)+h\left(\alpha_{2}\right)+\log 2$.

La proposition suivante quant à elle, ne découle pas immédiatement de la définition de la hauteur d'un point.

Proposition 2.2 (cf. [6, B.2.5]). - Soient $F_{0}, \ldots, F_{r}$ des polynômes homogènes de degré $d$. Soient $\phi=\left(F_{0}, \ldots, F_{r}\right)$ et $Z=Z\left(F_{0}, \ldots, F_{r}\right)$ le lieu géométrique commun des zéros des $F_{i}$.

1) Il existe $c$ tel que pour tout $x \notin Z$, on a $h(\phi(P)) \leq d h(P)+c$.

TOME $131-2003-\mathrm{N}^{\mathrm{O}} 2$ 
2) Soit $V$ une sous-variété fermée avec $V \cap Z=\varnothing$. Pour tout $P \in V(\overline{\mathbb{Q}})$,

$$
h(\phi(P))=d h(P)+O(1) .
$$

2.2. Propriétés du degré dynamique. - Nous commençons par justifier l'existence du degré dynamique.

LEMME 2.3 (cf. [15]). — Soit $\phi$ un automorphisme affine. La limite suivante existe et définit le degré dynamique :

$$
\delta(\phi):=\lim _{n \rightarrow \infty} \operatorname{deg}\left(\phi^{n}\right)^{1 / n}=\inf _{n \geq 1} \operatorname{deg}\left(\phi^{n}\right)^{1 / n} .
$$

Démonstration. - Si on pose $\delta:=\inf _{n \geq 1} \operatorname{deg}\left(\phi^{n}\right)^{1 / n}$ alors, pour tout $\varepsilon>0$ il existe $n_{0}$ tel que $\delta \leq \operatorname{deg}\left(\phi^{n_{0}}\right)^{1 / n_{0}} \leq \delta+\varepsilon$. Soit maintenant $n=q n_{0}+r$ la division euclidienne de $n$ par $n_{0}$; on a

$$
\operatorname{deg}\left(\phi^{n}\right)^{1 / n} \leq\left[\operatorname{deg}\left(\left(\phi^{n_{0}}\right)\right)^{q} \operatorname{deg}\left(\phi^{r}\right)\right]^{1 / n} \leq \operatorname{deg}\left(\phi^{n_{0}}\right)^{1 / n_{0}} c^{1 / n}
$$

où $c=\max _{0 \leq r \leq n_{0}-1} \operatorname{deg}\left(\phi^{r}\right)$. Ainsi $\delta \leq \operatorname{deg}\left(\phi^{n}\right)^{1 / n} \leq(\delta+\varepsilon) c^{1 / n}$ d'où $\lim \operatorname{deg}\left(\phi^{n}\right)^{1 / n}=\delta$.

Le degré dynamique est clairement invariant par conjugaison par un automorphisme affine. Le degré dynamique contrairement au degré algébrique n'est pas nécessairement un entier. En effet, $\phi(x, y, z)=\left(z+y^{2}, y+x^{3}, x\right)$ est de degré dynamique $\sqrt{6}$.

H. Bass et al. [1] ont montré une inégalité vérifiée par les degrés algébriques de $\phi$ et de $\phi^{-1}$.

Proposition 2.4 (cf. [1]). - Soit $\phi$ un automorphisme polynomial de $\mathbb{C}^{r}$, alors nous avons :

$$
\operatorname{deg}\left(\phi^{-1}\right) \leq(\operatorname{deg}(\phi))^{r-1}
$$

Cette proposition 2.4, nous permet d'obtenir la même inégalité pour les degrés dynamiques.

Lemme 2.5. - Soit $\phi$ un automorphisme polynomial de $\mathbb{C}^{r}$; alors

$$
\delta\left(\phi^{-1}\right) \leq(\delta(\phi))^{r-1} .
$$

Ce résultat est clair au vu de la définition et de la proposition 2.4. Il suffit en effet de remarquer que pour tout $n$, on a $\operatorname{deg}\left(\phi^{-n}\right) \leq\left(\operatorname{deg}\left(\phi^{n}\right)\right)^{r-1}$ ainsi que $\operatorname{deg}\left(\phi^{n}\right) \leq(\operatorname{deg}(\phi))^{n}$.

Nous constatons ainsi qu'une caractéristique de la dimension 2 est que tout automorphisme du plan affine a même degré que son inverse (que l'on considère le degré dynamique ou algébrique). De plus, nous pouvons ajouter grâce à la classification des automorphismes du plan affine que tous les automorphismes du plan affine ont un degré dynamique entier. En effet, le degré dynamique est invariant par conjugaison. 
Remarque 2.6. - Le degré dynamique est supérieur ou égal à 1. De plus, la propriété $\delta(\phi)=1$ n'implique pas $\operatorname{deg}(\phi)=1$. Considérons par exemple $\phi\left(X_{1}, X_{2}\right)=\left(X_{1}+X_{2}^{2}, X_{2}\right)$ : il s'agit d'un automorphisme affine de degré dynamique égal à 1 et de degré algébrique égal à 2 . Plus généralement, les applications triangulaires ainsi que leurs applications conjuguées sont de degré dynamique 1 . De même, l'application $\phi\left(X_{1}, X_{2}, X_{3}\right)=\left(X_{1}, X_{2}+X_{1}^{2}+X_{3}^{2}, X_{3}\right)$ est degré dynamique 1.

2.3. Degré dynamique et dynamique arithmétique. - Au vu de cette définition et de ces propriétés, il semble naturel d'essayer de relier le degré dynamique à la hauteur des itérés. En effet, nous avons le résultat suivant.

Proposition 2.7. - Soit $\phi$ un automorphisme de l'espace affine $\mathbb{A}^{r}$ et $P$ un point non-périodique de $\mathbb{A}^{r}(\overline{\mathbb{Q}})$.

- $\operatorname{Si} \delta(\phi)=1$, alors

$$
\lim _{B \rightarrow+\infty} \frac{N(\phi, P, B)}{\log B}=+\infty .
$$

- $S i \delta(\phi) \neq 1$, nous avons :

$\triangleright$ pour tout $P \in \mathbb{A}^{r}(\overline{\mathbb{Q}})$,

$$
\limsup _{n \rightarrow+\infty} \frac{\log h\left(\phi^{n}(P)\right)}{n} \leq \log \delta(\phi),
$$

$\triangleright$ pour tout point non-périodique $P \in \mathbb{A}^{r}(\overline{\mathbb{Q}})$ :

$$
\log B\left(\frac{1}{\log \delta(\phi)}+\frac{1}{\log \delta\left(\phi^{-1}\right)}+o(1)\right) \leq N(\phi, P, B) .
$$

Démonstration. - Soit $\varepsilon>0$, prenons $n_{0}$ tel que $\operatorname{deg}\left(\phi^{n_{0}}\right)^{1 / n_{0}} \leq \delta+\varepsilon$, alors $h\left(\phi^{n_{0}}(P)\right) \leq(\delta+\varepsilon)^{n_{0}} h(P)+c\left(n_{0}\right)$ et donc

$$
h\left(\phi^{q n_{0}}(P)\right) \leq(\delta+\varepsilon)^{q n_{0}}\left(h(P)+c^{\prime}\left(n_{0}\right)\right) .
$$

Par conséquent, si $n=q n_{0}+r$,

$$
h\left(\phi^{n}(P)\right)=h\left(\phi^{q n_{0}}\left(\phi^{r}(P)\right)\right) \leq(\delta+\varepsilon)^{q n_{0}}\left(h\left(\phi^{r}(P)\right)+c^{\prime}\left(n_{0}\right)\right) \ll(\delta+\varepsilon)^{n} .
$$

D'où le résultat.

Remarque 2.8. - Le résultat de la proposition peut aussi s'écrire : soient $\phi \in \operatorname{Aut}\left(\mathbb{A}^{r}\right), P$ un point non-périodique et $\varepsilon>0$. Il existe $n_{0} \in \mathbb{N} \backslash\{0\}$ tel que pour tout $n \geq n_{0}$, il existe $c_{1}(\varepsilon, \phi), c_{2}(\varepsilon, \phi)$ tels que,

$$
h\left(\phi^{n}(P)\right) \leq(\delta+\varepsilon)^{n}\left(c_{1}(\varepsilon, \phi) h(P)+c_{2}(\varepsilon, \phi)\right) .
$$

On peut se demander si on peut «enlever $\varepsilon$ ». L'exemple des automorphismes triangulaires, qui vérifient $\delta(\phi)=1$, montre que la réponse à cette question est négative. 
Proposition 2.9. - Le degré dynamique est invariant sur une classe de conjugaison. Ainsi, si $\phi$ est le conjugué d'un automorphisme régulier $\psi$ alors $\delta(\phi)=\operatorname{deg}(\psi)$ et $\delta\left(\phi^{-1}\right)=\operatorname{deg}\left(\psi^{-1}\right)$.

Démonstration. - Il existe $f \in \operatorname{Aut}\left(A^{r}\right)$ tel que $\phi=f^{-1} \psi f$. Pour tout $n \in \mathbb{N} \backslash\{0\}$, nous avons $\phi^{n}=f^{-1} \psi^{n} f$, d'où

$$
\operatorname{deg}\left(\phi^{n}\right) \leq \operatorname{deg}\left(f^{-1}\right)\left(\operatorname{deg}(\psi)^{n}\right) \operatorname{deg}(f) .
$$

Ainsi $\left(\operatorname{deg}\left(\phi^{n}\right)\right)^{1 / n} \leq\left(\operatorname{deg}\left(f^{-1}\right)\right)^{1 / n}\left(\operatorname{deg}(\psi)^{n}\right)^{1 / n}(\operatorname{deg}(f))^{1 / n} \operatorname{donc} \delta(\phi) \leq \delta(\psi)$. L'automorphisme $\psi$ étant conjugué à $\phi$, nous obtenons de manière analogue l'autre inégalité.

2.4. Généralités sur les automorphismes affines. - Nous donnons d'abord des propriétés générales puis, nous nous intéressons au cas des applications algébriquement stables (définies plus loin).

2.4.1. Premières propriétés. — Nous utiliserons à différentes reprises le lemme suivant :

LEMmE 2.10. - Soit $\phi$ un automorphisme non linéaire de $\mathbb{A}^{r}$. Alors on a

$$
\phi(H \backslash Z(\phi)) \subset Z\left(\phi^{-1}\right) \quad \text { et } \quad \phi^{-1}\left(H \backslash Z\left(\phi^{-1}\right)\right) \subset Z(\phi) .
$$

Une démonstration de ce lemme se trouve dans [15]; nous la rappelons cidessous.

Démonstration. - Soient $d \geq 2$ et $d^{\prime} \geq 2$ les degrés de $\phi$ et $\phi^{-1}$. Soient $\widetilde{\phi}$ et $\widetilde{\phi}^{-1}$ les applications rationnelles associées respectivement à $\phi$ et $\phi^{-1}$. Nous notons $F$ et $F^{-1}$ des relèvements à $\mathbb{A}^{r+1}$ de $\widetilde{\phi}$ et $\widetilde{\phi}^{-1}$. Nous avons alors :

$$
F \circ F^{-1}\left(x_{1}, \ldots, x_{r}, t\right)=F^{-1} \circ F\left(x_{1}, \ldots, x_{r}, t\right)=t^{d \cdot d^{\prime}-1}\left(x_{1}, \ldots, x_{r}, t\right) .
$$

D'où les inclusions

$$
\left.\phi(\{t=0\} \backslash Z(\phi)) \subset Z\left(\phi^{-1}\right) \quad \text { et } \quad \phi^{-1}\left(\{t=0\} \backslash Z\left(\phi^{-1}\right)\right) \subset Z(\phi)\right) .
$$

Le degré algébrique n'est en général pas stable par composition, en effet :

Proposition 2.11 (cf. [4]). — Soient $f, g$ deux applications rationnelles dominantes de $\mathbb{P}^{r}$ de degré algébrique respectif $d$ et $d^{\prime}$. Le degré algébrique de $f \circ g$ est égal à $d d^{\prime}$, si et seulement s'il n'existe pas d'hypersurface $V\left(V \subset \mathbb{P}^{r}\right)$ telle que $g(V \backslash Z(g)) \subset Z(f)$.

Ainsi pour un morphisme affine $f$ de degré $d$, le degré algébrique de $f^{2}$ n'est pas nécessairement égal à $d^{2}$. On définit donc la notion d'application algébriquement stable.

BULlETiN DE LA SOCIÉtÉ MATHÉMATIQUE DE FRANCE 


\subsubsection{Applications algébriquement stables}

DÉFInItion 2.12. - Une application rationnelle dominante $\phi$ de $\mathbb{P}^{r}$ est dite algébriquement stable s'il n'existe pas d'entier $n$ ni d'hypersurface $V$ tels que toutes les composantes de $\phi^{n}$ soient nulles sur $V$.

REMARQUE 2.13. - Si $\phi$ est une application algébriquement stable de degré $d$, alors, d'après la proposition 2.11, l'automorphisme $\phi^{n}$ est de degré $d^{n}$ pour tout entier $n$. Pour les applications algébriquement stables, les degrés algébrique et dynamique sont confondus (voir [15]). De plus, pour toute application algébriquement stable $\phi$, pour tout $m \leq n$ nous avons $Z\left(\phi^{m}\right) \subset Z\left(\phi^{n}\right)$.

Nous allons maintenant nous intéresser à la classification des automorphismes du plan affine.

2.5. Classification des automorphismes du plan affine. - En 1942, H.W.E. Jung [7] a obtenu une classification des automorphismes du plan affine. En 1989, ce résultat à été raffiné par S. Friedland et J. Milnor [5] en classifiant ces automorphismes à conjugaison près. Pour décrire ces résultats nous introduisons deux familles d'applications :

- une application de Hénon généralisée est une application $g$ de la forme

$$
g(x, y)=(p(x)-a y, x)
$$

où $a$ est une constante non nulle et $p$ est un polynôme unitaire de degré au moins 2 ;

- une application élémentaire est une application $e$ de la forme

$$
e(x, y)=(\alpha x+q(y), \beta y+\gamma)
$$

avec $\alpha, \beta, \gamma \in \mathbb{C}, \alpha \beta \neq 0$ et $q(y)$ une application polynomiale. Les applications élémentaires forment un groupe : appelé groupe des automorphismes élémentaires et noté $E$.

Dans la terminologie que nous utilisons les applications élémentaires sont des applications triangulaires, nous utiliserons, en dimension 2, indifféremment l'un de ces deux termes.

Remarque 2.14. - Une application de Hénon généralisée est un cas particulier d'automorphisme régulier.

THÉORÈme 2.15 (Friedland-Milnor [5]). — Soit $\phi$ un automorphisme polynomial de $\mathbb{C}^{2}$ alors, il existe $\psi \in \operatorname{Aut}\left(\mathbb{C}^{2}\right)$ tel que :

- soit $\psi^{-1} \phi \psi$ est la composée d'applications de Hénon généralisées,

- soit $\psi^{-1} \phi \psi$ est une application élémentaire.

Friedland et Milnor obtiennent également un résultat plus précis sur les applications élémentaires :

TOME $131-2003-\mathrm{N}^{\mathrm{O}} 2$ 
THÉORÈME 2.16 (cf. [5]). — Toute application élémentaire (i.e. tout élément de $E)$ est conjuguée à un automorphisme d'un des types suivants avec $\alpha, \beta \in \mathbb{C}$ :

a) $\phi_{a}(x, y)=(\alpha x, \beta y)$;

b) $\phi_{b}(x, y)=(\alpha x, y+1)$ ou $\phi_{b}^{\prime}(x, y)=(x+1, \beta y)$;

c) $\phi_{c}(x, y)=\left(\beta^{d}\left(x+y^{d}\right), \beta y\right)$, avec $d \in \mathbb{N} \backslash\{0\}$;

d) $\phi_{d}(x, y)=\left(\beta^{m}\left(x+y^{m} q\left(y^{r}\right)\right), \beta y\right)$ avec $\beta$ racine primitive $r$-ième de l'unité, $q$ un polynôme non constant, et $m \geq 0$.

Les applications $\phi_{a}, \phi_{b}, \phi_{b}^{\prime}, \phi_{c}$ et $\phi_{d}$ sont appelées applications élémentaires normalisées.

Remarque 2.17. - Ces résultats de classification sont encore vrais sur $\overline{\mathbb{Q}}$. En effet, si $\phi$ est défini sur $\overline{\mathbb{Q}}$ on obtient $\phi=\psi^{-1} \circ f \circ \psi$ avec $f, \psi \in \operatorname{Aut}\left(\mathbb{A}^{2}(\mathbb{C})\right)$ et $f$ est une application élémentaire ou une composée d'applications de Hénon généralisées. Les coefficients des polynômes définissant $\psi, \psi^{-1}$ et $f$ sont dans un corps de type fini $\mathbb{Q}\left(T_{1}, \ldots, T_{s}\right)$ (avec les $T_{i}$ non nécessairement algébriquement indépendants). Les polynômes se spécialisent en des polynômes à coefficients dans un corps de nombres $K$ (sauf pour $T$ dans un fermé propre de Zariski) et fournissent un automorphisme $f_{t}$ qui reste élémentaire (resp. un composé d'applications de Hénon généralisées) et un automorphisme $\psi_{t}$ d'où $\phi=\psi_{t}^{-1} \circ f_{t} \circ \psi_{t}$ avec $f_{t}, \psi_{t}$ définis sur $\overline{\mathbb{Q}}$.

L. Denis a utilisé ces applications élémentaires normalisées pour montrer que tout automorphisme du plan affine admet sur $k$ un nombre fini de points périodiques isolés.

LEMME 2.18. - Un produit fini d'applications de Hénon généralisées est un automorphisme régulier.

Ce résultat s'obtient directement en composant les applications.

\subsection{Propriétés des automorphismes réguliers}

2.6.1. Premières propriétés. — N. Sibony [15] donne une autre démonstration de la proposition 2.4 et la raffine dans le cas particulier des automorphismes réguliers.

Proposition 2.19 (cf. [15]). — Soit $\phi$ un automorphisme régulier de $A^{r}(\mathbb{C})$ de degré $d$, d'inverse $\phi^{-1}$ de degré $d^{\prime}$. On a

$$
d^{\ell}=d^{(r-\ell)} \quad \text { avec } \quad \ell:=\operatorname{dim}\left(Z\left(\phi^{-1}\right)\right)+1 .
$$

On a aussi $\operatorname{dim}(Z(\phi))+\operatorname{dim}\left(Z\left(\phi^{-1}\right)\right)=r-2$.

Ce résultat nous permet de constater qu'un automorphisme régulier ne peut avoir même degré que son inverse que si la dimension est paire, d'où le choix de la dimension 3 pour la construction de l'exemple suivant.

BULlETiN DE LA SOCIÉtÉ MATHÉMATIQUE DE FRANCE 
EXEMPLE 2.20. - Ainsi

$$
\phi\left(X_{1}, X_{2}, X_{3}\right)=\left(X_{1}+X_{2}^{2}+\left(X_{3}+X_{1}^{2}\right)^{2}, X_{2}+\left(X_{1}+X_{2}^{2}\right)^{2}, X_{3}+X_{1}^{2}\right)
$$

est un automorphime affine de degré 4 d'inverse l'application qui à $\left(X_{1}, X_{2}, X_{3}\right)$ associe

$$
\begin{gathered}
\left(X_{1}-X_{3}^{2}-\left(X_{2}-\left(X_{1}-X_{3}^{2}\right)^{2}\right)^{2}, X_{2}-\left(X_{1}-X_{3}^{2}\right)^{2}\right. \\
\left.X_{3}-\left[X_{1}-X_{3}^{2}-\left(X_{2}-\left(X_{1}-X_{3}^{2}\right)^{2}\right)^{2}\right]^{2}\right)
\end{gathered}
$$

de degré 16 avec $Z(\phi)=V\left(T, X_{1}, X_{2}\right)$ et $Z\left(\phi^{-1}\right)=V\left(T, X_{3}\right)$. C'est donc un automorphisme régulier, et dans ce cas $\ell=2$.

Nous serons amenée par la suite, à utiliser la proposition suivante démontrée par N. Sibony :

Proposition 2.21 (cf. [15]). - Soit $\phi$ un automorphisme régulier de degré d de $\mathbb{A}^{r}$. Alors, $\phi$ est algébriquement stable.

Cette proposition 2.21 se montre à partir du lemme 2.10.

Remarque 2.22. - On déduit immédiatement à l'aide du lemme 2.13 que si $\phi$ est un automorphisme régulier de degré $d$ alors $\phi^{n}$ est de degré $d^{n}$.

De manière naturelle, on peut s'interroger sur le comportement des lieux de non-définition des itérés par rapport à celui de l'automorphisme de départ.

\subsubsection{Stabilité par itération du caractère régulier}

Proposition 2.23. - Si $\phi$ est régulier de $\mathbb{A}^{r}$, alors pour tout entier naturel $n$ non nul $\phi^{n}$ est régulier et $Z\left(\phi^{n}\right)=Z(\phi)$.

Pour démontrer ce résultat, qui nous sera utile pour étudier la dynamique arithmétique des automorphismes réguliers, nous utiliserons le lemme suivant:

LEMME 2.24. - Si $\phi$ est un automorphisme régulier de $\mathbb{A}^{r}$, alors $\phi^{2}$ est régulier et $Z\left(\phi^{2}\right)=Z(\phi)$.

Démonstration. - Soit $P \in Z\left(\phi^{2}\right) \cap Z\left(\phi^{-2}\right)$, d'où $P \in\{T=0\}$. Nous avons $Z\left(\phi^{-1}\right) \subset Z\left(\phi^{-2}\right)$ et $Z(\phi) \subset Z\left(\phi^{2}\right)$. Si $P$ est dans l'hyperplan à l'infini et $P \notin Z(\phi)$, alors $\phi(P) \in \phi(T=0 \backslash Z(\phi)) \subset Z\left(\phi^{-1}\right)$; donc $\phi(P) \notin Z(\phi)$, et par conséquent $P \notin Z\left(\phi^{2}\right)$. Et par conséquent $\phi^{2}$ est régulier et comme $Z(\phi) \subset Z\left(\phi^{2}\right)$, nous avons $Z(\phi)=Z\left(\phi^{2}\right)$.

Le lemme 2.24 nous permet d'obtenir le résultat désiré (la proposition 2.23) pour les automorphismes réguliers.

TOME $131-2003-\mathrm{N}^{\mathrm{O}} 2$ 
Preuve de la proposition 2.23. - Du lemme 2.24, nous déduisons que pour tout entier $n$ l'automorphisme $\phi^{2^{n}}$ est régulier et $Z\left(\phi^{2^{n}}\right)=Z(\phi)$. De la proposition 2.21, nous déduisons que $\phi^{2^{n}}$ est algébriquement stable et donc que $Z\left(\phi^{m}\right) \subset Z\left(\phi^{2 m}\right)$. Nous avons ainsi $Z(\phi) \subset Z\left(\phi^{m}\right) \subset Z\left(\phi^{2^{m}}\right)=Z(\phi)$.

2.7. Conjugaison (par un automorphisme affine). — En dimension 2, la classification par classes de conjugaison des automorphismes affines est assez précise [5], il semble donc naturel de s'intéresser au comportement des points périodiques et non-périodiques sous l'action de la conjugaison.

La proposition suivante indique que, grosso modo, les automorphismes d'une même classe de conjugaison ont le même comportement arithmétique.

Proposition 2.25. - Soient $\psi, \phi$ des automorphismes de l'espace affine $\mathbb{A}^{r}$. Supposons qu'il existe un automorphisme $f$ de $\mathbb{A}^{r}$ tel que $\psi=f^{-1} \phi f$. Alors :

a) $P$ est $\psi$-périodique si et seulement si $f(P)$ est $\phi$-périodique.

b) Soit $P$ un point non- $\psi$-périodique. Pour $B$ assez grand, il existe $\eta, \eta^{\prime}>0$ tels que

$$
N\left(\psi, P, \frac{B}{\eta}\right) \leq N(\phi, f(P), B) \leq N\left(\psi, P, \eta^{\prime} B\right) .
$$

Le premier point a déjà été utilisé par L. Denis [2], pour étudier les points périodiques des automorphismes du plan affine.

Démonstration. - Le premier résultat est immédiat. Nous passons donc au second. Il existe $c, c^{\prime}, \eta, \eta^{\prime}$ tels que pour tout $P \in \mathbb{A}^{r}$ :

$$
h(f(P)) \leq \eta h(P)+c \quad \text { et } \quad h\left(f^{-1}(P)\right) \leq \eta^{\prime} h(P)+c^{\prime} .
$$

D'après la proposition 2.2 , nous pouvons prendre $\eta=\operatorname{deg} f$ et $\eta^{\prime}=\operatorname{deg} f^{-1}$ dans les inégalités précédentes.

Posons $Q=f(P)$. Alors si $h\left(\phi^{n}(Q)\right) \leq B$, nous avons

$$
h\left(f^{-1} \phi^{n} f(P)\right) \leq \eta^{\prime} B+c^{\prime},
$$

donc $N\left(P, \psi, \eta^{\prime} B+c^{\prime}\right) \geq N(f(P), \phi, B)$.

De manière analogue, si $h\left(f^{-1} \phi^{n} f(P)\right) \leq B^{\prime}$, on a $h\left(\phi^{n}(Q)\right) \leq \eta B^{\prime}+c$ et par conséquent

$$
N\left(f(P), \phi, \eta B^{\prime}+c\right) \geq N\left(P, \psi, B^{\prime}\right) .
$$

Prenons $B=\eta B^{\prime}+c$; alors

$$
N\left(P, \psi, \eta^{\prime} B+c^{\prime}\right) \geq N(f(P), \phi, B) \geq N\left(P, \psi, \frac{B}{\eta}+c_{1}\right) .
$$

Ainsi pour $B$ assez grand, on a :

$$
N\left(P, \psi, \eta^{\prime} B\right) \geq N(f(P), \phi, B) \geq N\left(P, \psi, \frac{B}{\eta}\right) .
$$

BULLETIN DE LA SOCIÉtÉ MATHÉMATIQUE DE FRANCE 
REMARQUE 2.26. - Les comportements des orbites stables par conjugaison sont les suivants :

$$
\log N(B) \gg \ll B, \quad N(B) \gg \ll B, \quad N(B) \sim c \log B .
$$

On ne peut pas obtenir de résultat plus précis pour l'ensemble d'une classe de conjugaison.

Dans une classe, l'estimation $\log N(B) \gg \ll B$ est bien la meilleure possible pour l'ensemble de la classe de conjugaison (et ce même en dimension 2). En effet, considérons l'application $f$ définie par $f(x, y)=(x, y+1)$ ainsi que son application conjuguée par l'application $g$ définie par $g(x, y)=(p(x)-a y, x)$ avec $a \neq 0$ and $p$ un polynôme de degré au moins 2. Nous obtenons alors :

$$
N(f, P, B) \sim c \exp B \quad \text { et } \quad N\left(g^{-1} \circ f \circ g, P, B\right) \sim c^{\prime} \exp \frac{B}{\operatorname{deg}(p)} .
$$

Ainsi, nous avons

$$
\frac{\log N\left(g^{-1} \circ f \circ g, P, B\right)}{\log N(f, P, B)} \sim \frac{1}{\operatorname{deg}(p)} \neq 1 .
$$

2.8. Conjugaison stable. - La notion de conjugaison stable intervient notamment dans la réduction de la conjecture jacobienne aux automorphismes de degré inférieur ou égal à 3 (voir [17] et [1]).

Nous définissons les applications de stabilisation puis, la notion de conjugaison stable.

Notation. - Soit $P=\left(x_{1}, \ldots, x_{t}\right) \in \mathbb{A}^{t}$. Pour $r \leq t$, nous noterons

$$
p_{r}: \mathbb{A}^{t} \longrightarrow \mathbb{A}^{r} \quad \text { avec } \quad p_{r}(P)=\left(x_{1}, \ldots, x_{r}\right) .
$$

DÉFInItion 2.27. - Soit $\phi$ un automorphisme de $\mathbb{A}^{r}$ défini par $\phi=$ $\left(F_{1}, \ldots, F_{r}\right)$ avec $F_{i} \in k\left[X_{1}, \ldots, X_{r}\right]$ pour tout $1 \leq i \leq n$. Pour tout $t \in \mathbb{N} \backslash\{0\}$, notons $\phi^{[t]}$ l'automorphisme de $\mathbb{A}^{r+t}$ suivant :

$$
\phi^{[t]}\left(x_{1} \ldots, x_{r}, y_{1}, \ldots, y_{t}\right)=\left(F_{1}(x), \ldots, F_{r}(x), y_{1}, \ldots, y_{t}\right) .
$$

L'application $\phi^{[t]}$ est une application de stabilisation associée à $\phi$.

L'application $\phi^{[t]}$ définie ci-dessus est clairement un automorphisme.

DÉFInItion 2.28. - Soient $\phi$ un automorphisme de $\mathbb{A}^{r}$ et $\psi$ un automorphisme de $\mathbb{A}^{s}$. S'il existe $t \in \mathbb{N}$, avec $t \geq r, t \geq s$ tel que $\phi^{[t-r]}$ et $\psi^{[t-s]}$ soient conjugués dans $\operatorname{Aut}\left(\mathbb{A}^{t}\right)$, alors $\phi$ et $\psi$ sont dits stablement conjugués.

Nous nous intéressons au lien entre la dynamique arithmétique d'un automorphisme affine et la dynamique arithmétique d'un automorphisme qui lui est stablement conjugué.

TOME $131-2003-\mathrm{N}^{\mathrm{O}} 2$ 
LEMme 2.29. - Soit $\phi$ un automorphisme de $\mathbb{A}^{r}, \phi^{[t]}$ avec $1 \leq t$ une application de stabilisation associée à $\phi$. Nous avons alors :

- $\operatorname{Per}\left(\phi^{[t]}\right)=\operatorname{Per}(\phi) \times \mathbb{A}^{t}$.

- Si $P=(x, y)$ est un point non-périodique de $\phi_{t}$, alors il existe c tel que

$$
N\left(\phi, p_{r}(P), B-c\right) \leq N\left(\phi^{[t]}, P, B\right) \leq N\left(\phi, p_{r}(P), B\right)
$$

La démonstration de ce lemme est claire au vu de la définition. Si $P=(x, y)$ est un point non-périodique de $\phi_{t}$, alors il existe $c_{1}(y)$ tel que

$$
h\left(\phi^{n}(x)\right) \leq h\left(\left(\phi^{[t]}\right)^{n}(P)\right) \leq h\left(\phi^{n}(x)\right)+c_{1}(y)
$$

d'où le résultat suivant sur la taille des orbites des points non-périodiques.

REMARQUe 2.30. - La remarque concernant les classes de conjugaisons est encore valable ici. Les comportements stables des orbites pour $\phi$ variant dans une classe de conjugaison sont les suivants :

$$
\log N(B) \gg \ll B, \quad N(B) \gg \ll B, \quad N(B) \sim c \log B
$$

On ne peut pas obtenir de résultat plus précis pour l'ensemble d'une classe de conjugaison.

Corollaire 2.31. - Soit $\phi \in \operatorname{Aut}\left(\mathbb{A}^{r}(k)\right), \psi \in \operatorname{Aut}\left(\mathbb{A}^{s}(k)\right)$ deux automorphismes stablement conjugués dans $\operatorname{Aut}\left(\mathbb{A}^{t}(k)\right)$. Alors :

- L'automorphisme $\phi$ admet un nombre fini de points périodiques isolés sur $k$ si et seulement si $\psi$ admet un nombre fini de points périodiques isolés sur $k$.

- Les tailles des orbites des points non-périodiques sont de même nature.

La démonstration de ce corollaire utilise essentiellement la proposition 2.25 sur les automorphismes conjugués.

\section{Automorphismes réguliers}

Le principal résultat de cette partie, qui est une amélioration du résultat que nous avons montré dans [11] est le théorème A. Son corollaire, le corollaire B, est obtenu à l'aide de la proposition 2.25.

La démonstration du théorème $\mathrm{A}$, se décompose essentiellement en deux étapes, la première est l'obtention d'une inégalité à l'aide des propriétés de bases sur les hauteurs et la deuxième est purement combinatoire.

BULLETIN DE LA SOCIÉtÉ MATHÉMATIQUE DE FRANCE 


\subsection{Un résultat préliminaire}

THÉORÈme 3.1. - Soit $\psi_{1}, \ldots, \psi_{n}: \mathbb{A}^{s} \rightarrow \mathbb{A}^{r}$ des applications polynomiales de degré $d_{1}, \ldots, d_{n}$. Soit $Z\left(\psi_{i}\right)$ le lieu de non-définition de l'application rationnelle $\widetilde{\psi}_{i}$ associée à $\psi_{i}$. Supposons,

$$
Z\left(\psi_{1}\right) \cap Z\left(\psi_{2}\right) \cap \cdots \cap Z\left(\psi_{n}\right)=\varnothing \quad \text { avec } \quad \widetilde{\psi}_{i}: \mathbb{P}^{s} \rightarrow \mathbb{P}^{r} .
$$

Alors, il existe une constante $c$ (ne dépendant que de $\left.\psi_{1}, \ldots, \psi_{n}\right)$ telle que pour tout point $P \in \mathbb{A}^{s}(\overline{\mathbb{Q}})$,

$$
\sum_{i=1}^{r} \frac{1}{d_{i}} h\left(\psi_{i}(P)\right) \geq h(P)-c .
$$

REMARQUE 3.2. - Nous utiliserons essentiellement ce théorème sous la forme suivante. Soit $d=\min _{i} d_{i}$. Il existe $C$ tel que, pour tout point $P \in \mathbb{A}^{s}(\overline{\mathbb{Q}})$,

$$
\sum_{i=1}^{r} h\left(\psi_{i}(P)\right) \geq d h(P)-C .
$$

Démonstration. - La preuve repose essentiellement sur des propriétés de bases des hauteurs. Soient $D=d_{1} d_{2} \cdots d_{n}$ et $D_{i}=D / d_{i}$. Nous écrirons les applications rationnelles $\widetilde{\psi}_{i}=\left(\widetilde{F}_{i, 1}, \ldots, \widetilde{F}_{i, r+1}\right)$, avec $\widetilde{F}_{i, j} \in \overline{\mathbb{Q}}\left(x_{1}, \cdots, x_{s+1}\right)$ polynôme homogène de degré $d_{i}$ pour $1 \leq i \leq n, 1 \leq j \leq r+1$ et $\widetilde{F}_{i, r+1}=x_{s+1} d_{i}$.

Considérons l'application

$$
\Psi=\left[\widetilde{F}_{1,1}^{D_{1}}, \ldots, \tilde{F}_{1, r+1}^{D_{1}}, \ldots, \widetilde{F}_{n, 1}^{D_{n}}, \ldots, \tilde{F}_{n, r+1}^{D_{n}}\right] .
$$

Par construction, $\Psi: \mathbb{P}^{s} \rightarrow \mathbb{P}^{n(r+1)-1}$ est une application rationnelle et l'hypothèse $Z\left(\psi_{1}\right) \cap Z\left(\psi_{2}\right) \cap \cdots \cap Z\left(\psi_{n}\right)=\varnothing$ implique que $\Psi$ est un morphisme. Par conséquent, d'après la proposition 2.2 , pour tout $P \in \mathbb{P}^{s}(\overline{\mathbb{Q}})$,

$$
h(\Psi(P))=D h(P)+O(1) .
$$

Soit $P=\left(x_{1}, \ldots, x_{s}, 1\right)$; pour tout $v \in M_{k}$, nous avons

$$
\max _{i, j}\left|F_{i, j}^{D_{i}}(P)\right|_{v} \leq \prod_{i=1}^{n} \max _{j}\left|F_{i, j}^{D_{i}}(P)\right|_{v} .
$$

Dans chaque expression de la forme $\max _{j}\left|F_{i, j}^{D_{i}}(P)\right|_{v}$, le maximum tient compte du terme $\left|F_{i, r+1}(P)\right|_{v}=1$. En revenant aux notations de départ, nous obtenons

$$
|\Psi(P)|_{v} \leq \prod_{i=1}^{n}\left|\psi_{i}(P)\right|_{v}^{D_{i}}
$$

TOME $131-2003-\mathrm{N}^{\mathrm{O}} 2$ 
en prenant le produit sur toutes les places avec les puissances adéquates, nous obtenons l'inégalité

$$
H(\Psi(P)) \leq \prod_{i=1}^{n} H\left(\psi_{i}(P)\right)^{D_{i}} .
$$

D’où le résultat désiré, par passage au logarithme dans l'inégalité $\left.{ }^{* *}\right)$, par substitution dans la relation $(*)$, puis en divisant par $D$.

\subsection{Lemmes de comptages}

3.2.1. Minoration. - Ce lemme est directement inspiré des articles de J. Silverman [16] et L. Denis [2].

LEMME 3.3. - Soit $\phi$ un automorphisme régulier avec

$$
\operatorname{dm}:=\min \left(\operatorname{deg}(\phi), \operatorname{deg}\left(\phi^{-1}\right)\right)>2 .
$$

Alors, il existe $c>0$ tel que pour tout point $P \in \mathbb{A}^{r}$, pour tout $n \in \mathbb{N} \backslash\{0\}$ :

$$
h\left(\phi^{n}(P)\right)+h\left(\phi^{-n}(P)\right) \geq\left(a-a^{-1}\right)\left(a^{n}+a^{-n}\right)\left(h(P)-\frac{c}{\mathrm{dm}-2}\right),
$$

avec $a=\frac{1}{2}\left(\mathrm{dm}+\left(\mathrm{dm}^{2}-4\right)^{\frac{1}{2}}\right)$.

Démonstration. - La suite de terme général

$$
b_{n}=\frac{\sqrt{\mathrm{dm}^{2}-4}}{\mathrm{dm}^{2}-4}\left(a^{n}-a^{-n}\right)
$$

avec $a=\frac{1}{2}\left(\mathrm{dm}+\sqrt{\mathrm{dm}^{2}-4}\right), b_{0}=0$ et $b_{1}=1$ est une suite à termes positifs qui vérifie

$$
b_{i+1}-\mathrm{dm} \cdot b_{i}+b_{i-1}=0 \quad \text { pour tout } i \geq 1 .
$$

Introduisons la notation

$$
H_{n}:=h\left(\phi^{n}(P)\right)+h\left(\phi^{-n}(P)\right)-\frac{2 c}{\mathrm{dm}-2} .
$$

Nous appliquons le théorème 3.1 à $\phi$ et à $\phi^{-1}$; plus précisément, nous utilisons la forme plus faible de la remarque 3.2. Il existe $c>0$ tel que pour tout $P \in \mathbb{A}^{r}(\overline{\mathbb{Q}}):$

$$
h(\phi(P))+h\left(\phi^{-1}(P)\right) \geq \operatorname{dm} \cdot h(P)-c .
$$

De plus, en considérant $P=\phi^{i}(Q)$, puis $P=\phi^{-i}(Q)$ avec $i \in \mathbb{N} \backslash\{0\}$ dans la relation (1), nous avons

$$
\begin{gathered}
h\left(\phi^{i+1}(Q)\right)+h\left(\phi^{i-1}(Q)\right) \geq \mathrm{dm} \cdot h\left(\phi^{i}(Q)\right)-c, \\
h\left(\phi^{-i+1}(Q)\right)+h\left(\phi^{-i-1}(Q)\right) \geq \mathrm{dm} \cdot h\left(\phi^{-i}(Q)\right)-c,
\end{gathered}
$$

d'où par addition

$$
H_{i+1} \geq \mathrm{dm} \cdot H_{i}-H_{i-1} \quad \text { pour tout } i \geq 1 .
$$

BULLETIN DE LA SOCiÉTÉ MATHÉMATIQUe DE FRANCE 
De plus, d'après la condition (1) nous avons $H_{1} \geq \frac{1}{2} \mathrm{dm} \cdot H_{0}$.

Considérons maintenant :

$$
0 \leq \sum_{i=1}^{n} b_{n-i}\left(H_{i+1}-\mathrm{dm} \cdot H_{i}+H_{i-1}\right)=H_{n}+b_{n-1} H_{0}-b_{n} H_{1}
$$

ou encore $\left(\frac{1}{2} \mathrm{dm} \cdot b_{n}-b_{n-1}\right) H_{0} \leq H_{n}$.

De plus, comme on a $a+a^{-1}=\operatorname{dm}$ et $a+a^{-1}=\sqrt{\mathrm{dm}^{2}-4}$, en remplaçant $b_{n}$ et $b_{n-1}$ par leurs expressions, nous obtenons l'égalité

$$
\operatorname{dm} \cdot b_{n}-2 b_{n-1}=a^{n}-a^{-n} \geq\left(a-a^{-1}\right)\left(a^{n}+a^{-n}\right),
$$

d'où le résultat.

3.2.2. Spécificité des points non-périodiques. — Nous montrerons tout d'abord la croissance à partir d'un certain rang de la suite des hauteurs des itérés d'un point non-périodique.

Proposition 3.4. - Soient $\phi$ un automorphisme régulier de $\mathbb{A}^{r}$ et $P$ un point non-périodique. Alors les suites $\left(h\left(\phi^{k r}(P)\right)\right)_{k \in \mathbb{N}}$ et $\left(h\left(\phi^{-k r}(P)\right)\right)_{k \in \mathbb{N}}$ sont croissantes à partir d'un certain rang.

Remarque 3.5. - Si nous appliquons le théorème 3.1 à $\phi$ et $\phi^{-1}$, nous arrivons à montrer un peu mieux, à savoir, la croissance de $h\left(\phi^{n}(P)\right)$ et $h\left(\phi^{-n}(P)\right)$ à partir d'un certain rang; mais la proposition 3.4 est suffisante pour le résultat que nous voulons démontrer.

Démonstration. - Grâce au lemme 2.23, pour tout entier a non nul, nous avons $Z\left(\phi^{a \ell}\right) \cap Z\left(\phi^{-a(r-\ell)}\right)=\varnothing$. Nous pouvons donc appliquer le théorème 3.1 à $\phi^{a \ell}$ et à $\phi^{-a(r-\ell)}$. D'après les propositions $2.21,2.19$ et la remarque 2.13, nous avons également $\operatorname{deg}\left(\phi^{a \ell}\right)=\operatorname{deg}\left(\phi^{-a(r-\ell)}\right)=d^{a \ell}$.

Nous considérons l'inégalité (1). Nous obtenons alors : pour tout entier $a$ et pour tout point $P$ de l'espace affine,

$$
h\left(\phi^{a \ell}(P)\right)+h\left(\phi^{-a(r-\ell)}(P)\right) \geq d^{a \ell} h(P)-c_{a} .
$$

Supposons la conclusion de la proposition fausse. Alors, pour tout $m_{0}>1$, il existe $m>m_{0}$ tel que

$$
h\left(\phi^{r m}(P)\right) \geq h\left(\phi^{r(m-1)}(P)\right) \quad \text { et } \quad h\left(\phi^{r m}(P)\right) \geq h\left(\phi^{r(m+1)}(P)\right) .
$$

Considérons l'inégalité $(2)$ au point $\phi^{r(m+1)-a \ell}(P)$. Nous obtenons alors :

$$
h\left(\phi^{r(m+1)}(P)\right)+h\left(\phi^{r(m+1)-a r}(P)\right) \geq d^{a \ell} h\left(\phi^{r(m+1)-a \ell}(P)\right)-c_{a} .
$$

Pour $a=2$, nous avons,

$$
h\left(\phi^{r(m+1)}(P)\right)+h\left(\phi^{r(m-1)}(P)\right) \geq d^{2 \ell} h\left(\phi^{r(m+1)-2 \ell}(P)\right)-c_{2},
$$

TOME $131-2003-\mathrm{N}^{\mathrm{O}} 2$ 
ainsi,

$$
\begin{aligned}
2 h\left(\phi^{r m}(P)\right) & \geq h\left(\phi^{r(m+1)}(P)\right)+h\left(\phi^{r(m-1)}(P)\right) \\
& \geq d^{2 \ell} h\left(\phi^{r(m+1)-2 \ell}(P)\right)-c_{2} .
\end{aligned}
$$

Or, $\phi^{2 \ell-r}$ est un morphisme de degré $d^{2 \ell-r}$ si $2 \ell \geq r$ ou de degré $d^{2 \ell-r}$ si $2 \ell<r$.

$$
\begin{aligned}
h\left(\phi^{r m}(P)\right) & =h\left(\phi^{2 \ell-r}\left(\phi^{r(m+1)-2 \ell}(P)\right)\right) \\
& \leq\left\{\begin{array}{lll}
d^{2 \ell-r}\left[h\left(\phi^{r(m+1)-2 \ell}(P)\right)+c\right] & \text { si } & 2 \ell \geq r, \\
d^{\prime r-2 \ell}\left[h\left(\phi^{r(m+1)-2 \ell}(P)\right)+c\right] & \text { si } & 2 \ell<r .
\end{array}\right.
\end{aligned}
$$

Nous avons ainsi

$$
\left\{\begin{array}{l}
d^{2 \ell} \leq 2 d^{2 \ell-r} \Longrightarrow d^{r} \leq 2 \\
d^{2 \ell} \leq 2 d^{\prime r-2 \ell} \Longleftrightarrow d^{\prime 2(r-\ell)} \leq 2 d^{\prime r-2 \ell} \Longrightarrow d^{\prime r} \leq 2 .
\end{array}\right.
$$

D'où le résultat.

Nous utiliserons, essentiellement le résultat suivant, pour déterminer un équivalent asymptotique de la suite :

THÉORÈme 3.6. - Soit $\phi$ un automorphisme régulier de degré d d'inverse de degré $d^{\prime}$. Pout tout point non-périodique $P$, nous avons

$$
\lim _{n \rightarrow+\infty} \frac{\log \left(h\left(\phi^{n}(P)\right)\right.}{n}=\log d \quad \text { et } \quad \lim _{n \rightarrow+\infty} \frac{\log \left(h\left(\phi^{-n}(P)\right)\right.}{n}=\log d^{\prime} .
$$

Pour démontrer ce théorème tout comme pour montrer le caractère fini du nombre de points périodiques d'un automorphisme régulier sur $k$, nous utilisons le lemme technique suivant.

Lemme 3.7. - Soit $\phi$ un automorphisme régulier de degré $d$. Pour tout point non-périodique $P$ de l'espace affine, pour tout $a \in \mathbb{N} \backslash\{0\}$, il existe $m_{0} \in \mathbb{N}$ tel que pour tous entiers $m, s$ vérifiant mal $+s \geq m_{0}+$ ar, alors

$$
h^{\prime}\left(\phi^{a m \ell+s}(P)\right) \geq\left(\frac{d^{a \ell}}{2}\right)^{m} h^{\prime}\left(\phi^{s}(P)\right),
$$

avec $h^{\prime}(P)=h(P)-c_{a} /\left(d^{a \ell}-2\right)$.

Démonstration. - En appliquant la relation $(2)$ à $\phi^{n-a \ell}(P)$, nous obtenons

$$
h\left(\phi^{n}(P)\right)+h\left(\phi^{n-a r}(P)\right) \geq d^{a \ell} h\left(\phi^{n-a \ell}(P)\right)-c_{a} .
$$

Cette relation est valable pour tout point $P$ de l'espace affine. Nous considérons maintenant un point non périodique $P$. D'après la proposition 3.4 , pour tout point non-périodique $P$, il existe $m_{0}(P)=m_{0}$ tel que pour tout $m \geq m_{0}$, la suite de terme général $h\left(\phi^{r m}(P)\right)$ est croissante. 
Ainsi nous avons $h\left(\phi^{n}(P)\right) \geq h\left(\phi^{n-a r}(P)\right)$ pour $n-a r \geq m_{0}$. Donc

$$
2 h\left(\phi^{n}(P)\right) \geq h\left(\phi^{n}(P)\right)+h\left(\phi^{n-a r}(P)\right) \geq d^{a \ell} h\left(\phi^{n-a \ell}(P)\right)-c_{a} .
$$

Nous posons maintenant $n=m a \ell+s$ et définissons la hauteur $h^{\prime}$ par $h^{\prime}(P)=h(P)-c_{a} /\left(d^{a \ell}-2\right)$. Nous avons ainsi

$$
h^{\prime}\left(\phi^{a m \ell+s}(P)\right) \geq \frac{d^{a \ell}}{2} h^{\prime}\left(\phi^{a(m-1) \ell+s}(P)\right)
$$

et par conséquent

$$
h^{\prime}\left(\phi^{a m \ell+s}(P)\right) \geq\left(\frac{d^{a \ell}}{2}\right)^{m} h^{\prime}\left(\phi^{s}(P)\right) .
$$

Démonstration du théorème 3.6. - Grâce à cette relation nous montrons, dans un premier temps, l'inégalité

$$
\liminf _{n \rightarrow+\infty} \frac{\log h\left(\phi^{n}(P)\right)}{n} \geq \log d .
$$

En effet,

$$
\begin{aligned}
\frac{\log h^{\prime}\left(\phi^{a m \ell+s}(P)\right)}{a m \ell} & \geq \frac{m\left(\log d^{a \ell}-\log 2\right)+\log h^{\prime}\left(\phi^{s}(P)\right)}{a m \ell} \\
& \geq \frac{a m \ell \log d}{a m \ell}-\frac{\log 2}{a \ell}+o\left(\frac{1}{m}\right) \\
& =\log d-\frac{\log 2}{a \ell}+o\left(\frac{1}{m}\right) .
\end{aligned}
$$

Donc $\liminf _{m, a \rightarrow+\infty} \frac{\log h\left(\phi^{a m \ell+s}(P)\right)}{a m \ell} \geq \log d$. Or,

$$
\begin{aligned}
\liminf _{n \rightarrow+\infty} \frac{\log h\left(\phi^{n}(P)\right)}{n} & =\liminf _{m, a \rightarrow+\infty} \frac{\log h\left(\phi^{a m \ell+s}(P)\right)}{a m \ell+s} \\
& =\liminf _{m, a \rightarrow+\infty} \frac{\log h\left(\phi^{a m \ell+s}(P)\right)}{a m \ell} \geq \log d,
\end{aligned}
$$

donc

$$
\liminf _{n \rightarrow+\infty} \frac{\log h\left(\phi^{n}(P)\right)}{n} \geq \log d
$$

De plus $\phi^{n}$ est un morphisme de degré $d^{n}$, donc pour tout point $P \in \mathbb{A}^{r}$, nous avons

$$
h\left(\phi^{n}(P)\right) \leq d^{n}(h(P)+O(1)) .
$$

D'où le résultat désiré. Nous obtenons de manière analogue le résultat pour l'application $\phi^{-1}$. 
3.3. Démonstration du théorème $\mathbf{A}$. - Nous sommes maintenant en mesure de démontrer le théorème sur les automorphismes réguliers. Nous considérons tout d'abord le cas des points périodiques puis, celui des points non-périodiques.

Démonstration du théorème $A$. - Soit $\phi$ un automorphisme régulier vérifiant $\min \left(\operatorname{deg}(\phi), \operatorname{deg}\left(\phi^{-1}\right)\right)>2$. Le premier point résulte d'une simple application du lemme 3.3. D'après le lemme 3.3,

$$
h\left(\phi^{n}(P)\right)+h\left(\phi^{-n}(P)\right) \geq\left(a-a^{-1}\right)\left(a^{n}+a^{-n}\right)\left(h(P)-\frac{c}{\mathrm{dm}-2}\right)+\frac{2 c}{\mathrm{dm}-2} .
$$

Soit $P$ un point périodique. Il existe $n \in \mathbb{N}$ tel que $\phi^{n}(P)=P$; ainsi

$$
\frac{c}{\mathrm{dm}-2}\left[\left(a-a^{-1}\right)\left(a^{n}+a^{-n}\right)-2\right] \geq h(P)\left[\left(a-a^{-1}\right)\left(a^{n}+a^{-n}\right)-2\right],
$$

d'où le résultat.

Si $\min \left(\operatorname{deg}(\phi), \operatorname{deg}\left(\phi^{-1}\right)\right)=2$, il suffit d'appliquer le lemme 3.3 à $\phi^{2}$.

Comme $\phi$ est un automorphisme régulier (de degré $d$ d'inverse de degré $d^{\prime}$ ), d'après le théorème 3.6 , pour $n>n_{0}$ pour tout point $P$ non-périodique nous avons

$$
\lim _{n \rightarrow+\infty} \frac{\log \left(h\left(\phi^{n}(P)\right)\right.}{n}=\log d \quad \text { et } \quad \lim _{n \rightarrow+\infty} \frac{\log \left(h\left(\phi^{-n}(P)\right)\right.}{n}=\log d^{\prime} .
$$

Nous avons ainsi, pour tout $n \geq n_{0}$,

$$
(1-\varepsilon) n \log d \leq \log h\left(\phi^{n} P\right) \leq(1+\varepsilon) n \log d
$$

et de la même manière, pour tout $n \geq n_{0}$,

$$
(1-\varepsilon) n \log d^{\prime} \leq \log h\left(\phi^{-n} P\right) \leq(1+\varepsilon) n \log d^{\prime} .
$$

En utilisant les fontions

$$
\begin{aligned}
& N^{+}(B)=\#\left\{n \in \mathbb{N} \mid \log \left(h\left(\phi^{n} P\right)\right) \leq \log B\right\}, \\
& N^{-}(B)=\#\left\{n \in \mathbb{N} \mid \log \left(h\left(\phi^{-n} P\right)\right) \leq \log B\right\},
\end{aligned}
$$

nous obtenons

$$
\frac{\log B}{1+\varepsilon}\left(\frac{1}{\log d}+\frac{1}{\log d^{\prime}}\right)-2 n_{0}-2 \leq N(B) \leq \frac{2 \log B}{1-\varepsilon}\left(\frac{1}{\log d}+\frac{1}{\log d^{\prime}}\right)+1 .
$$

Donc,

$$
N(\phi, P, B) \sim\left(\frac{1}{\log d}+\frac{1}{\log d^{\prime}}\right) \log B .
$$

Or on a $d^{\ell}=d^{\prime r-\ell}$ d'après la proposition 2.19; ainsi

$$
\frac{1}{\log d}+\frac{1}{\log d^{\prime}}=\frac{r}{\ell} \frac{1}{\log d}
$$

d'où le résultat.

BULletin DE LA SOCiÉtÉ MATHÉmATIQUE DE FRANCE 
La démonstration du corollaire $\mathrm{B}$ repose essentiellement sur le théorème $\mathrm{A}$ et sur la proposition 2.25 qui concerne les classes de conjugaison. Plus précisément, nous utilisons également la relation $(* * *)$, la remarque 2.22 qui précise que les degrés dynamique et algébrique d'un automorphisme régulier sont égaux et l'invariance par conjugaison du degré dynamique.

Nous nous intéressons maintenant aux applications triangulaires qui jouent un rôle important dans les travaux sur la classification des automorphismes affines. En effet, en dimension 2, H. Jung [7] et W. van der Kulk [9] ont montré que le groupe des automorphismes du plan affine complexe pouvait s'écrire comme le produit amalgamé des applications linéaires avec les applications triangulaires (également appelées applications de Jonquières). Pour plus de précisions sur les automorphismes affines nous renvoyons le lecteur à [3].

\section{Applications triangulaires}

Notation. - Nous noterons $\mu^{\infty}$ l'ensemble des racines de l'unité.

L'inverse d'un automorphisme triangulaire est un automorphisme triangulaire; il est de la forme

$$
\phi^{-1}\left(X_{1}, \ldots, X_{r}\right)=\left(b_{1} X_{1}+Q_{1}\left(X_{2}, \ldots, X_{r}\right), \ldots, b_{r} X_{r}+Q_{r}\right)
$$

avec $Q_{i} \in k\left[X_{i+1}, \ldots, X_{r}\right]$ pour $1 \leq i \leq r-1$ et $Q_{r} \in \overline{\mathbb{Q}}, b_{i} \in \overline{\mathbb{Q}}^{*}$ pour $1 \leq i \leq r$. Plus précisément, nous avons $b_{i}=1 / a_{i}$. Ainsi, si $a_{i} \in \mu^{\infty}$ alors $b_{i} \in \mu^{\infty}$ et si de plus $a_{i}=1$, nous avons également $b_{i}=1$. Ces remarques sont assez importantes car, comme nous le verrons plus loin, le comportement dynamique des applications triangulaires repose essentiellement sur la nature des coefficients $a_{i}$ et $b_{i}$. Les applications triangulaires sont de degré dynamique 1 . En effet, il existe $D \in \mathbb{N}$ tel que pour tout $n \in \mathbb{N}, \operatorname{deg}\left(\phi^{n}\right) \leq D$.

Dans la démonstration du théorème $\mathrm{C}$, nous utiliserons les propriétés des hauteurs rappelées en 2.1.

Démonstration du théorème $C$. — Soit

$$
\phi\left(X_{1}, \ldots, X_{r}\right)=\left(a_{1} X_{1}+F_{1}\left(X_{2}, \ldots, X_{r}\right), \ldots, a_{r} X_{r}+F_{r}\right) .
$$

Pour tout $n \geq 1$ nous notons

$$
\phi^{n}\left(X_{1}, \ldots, X_{r}\right)=\left(a_{1}^{n} X_{1}+F_{1}^{(n)}\left(X_{2}, \ldots, X_{r}\right), \ldots, a_{r}^{n} X_{r}+F_{r}^{(n)}\right)
$$

avec

$$
\begin{aligned}
F_{i}^{(n)}\left(X_{i+1}, \ldots, X_{r}\right)= & a_{i} F_{i}^{(n-1)}\left(X_{i+1}, \ldots, X_{r}\right) \\
& +F_{i}\left(a_{i+1}^{n-1} X_{i+1}+F_{i+1}^{(n-1)}\left(X_{i+2}, \ldots, X_{r}\right)\right. \\
& \left.\ldots, a_{r}^{n-1} X_{r}+F_{r}^{(n-1)}\right)
\end{aligned}
$$

et $F_{r}^{(n)}=n F_{r}$. 
On peut aisément déduire de ces relations qu'une application triangulaire admet un nombre fini de points périodiques isolés sur $k$.

Nous nous intéressons maintenant aux points non-périodiques. Deux cas se distinguent suivant les valeurs des $a_{i}$.

Si on a $a_{i} \in \mu^{\infty}$ pour tout $i$, on peut supposer $a_{i}=1$ quitte à remplacer $\phi$ par une puissance de $\phi$. Supposons $a_{1}=\cdots=a_{r}=1$. Comme $F_{r}^{(n)}=n F_{r}$, on a donc $h\left(F_{r}^{(n)}(P)\right) \leq \log |n|+C$.

Supposons que $h\left(F_{j}^{(n)}\left(x_{j+1}, \ldots, x_{r}\right)\right) \leq \log |n|+c^{\prime}$ a déjà été prouvé pour $j=i+1, \ldots, r$. Alors :

$$
\begin{aligned}
& h\left(F_{i}^{(n)}\left(x_{i+1}, \ldots, x_{r}\right)\right) \\
& =h\left(a_{i} F_{i}^{(n-1)}\left(x_{i+1}, \ldots, x_{r}\right)\right. \\
& \left.\quad \quad+F_{i}\left(a_{i+1} x_{i+1}+F_{i+1}^{(n-1)}\left(x_{i+2}, \ldots, x_{r}\right), \ldots, a_{r} x_{r}+F_{r}^{(n-1)}\right)\right) \\
& \leq h\left(a_{i} F_{i}^{(n-1)}\left(x_{i+1}, \ldots, x_{r}\right)\right) \\
& \quad+h\left(F_{i}\left(a_{i+1} x_{i+1}+F_{i+1}^{(n-1)}\left(x_{i+2}, \ldots, x_{r}\right), \ldots, a_{r} x_{r}+F_{r}^{(n-1)}\right)\right) \\
& \quad+\log 2 \\
& \leq c \log |n| \\
& \quad+c^{\prime} h\left(a_{i+1} x_{i+1}+F_{i+1}^{(n-1)}\left(x_{i+2}, \ldots, x_{r}\right), \ldots, a_{r} x_{r}+F_{r}^{(n-1)}\right) \\
& \quad+c^{\prime \prime} \\
& \leq c \log |n|+c^{\prime} h\left(a_{i+1} x_{i+1}, \ldots, a_{r} x_{r}\right) \\
& \quad+c^{\prime} h\left(F_{i+1}^{(n-1)}\left(x_{i+2}, \ldots, x_{r}\right), \ldots, F_{r}^{(n-1)}\right)+c_{3} \\
& \leq \quad \\
& c_{4} \log |n|+c_{5} .
\end{aligned}
$$

Si $F_{r} \neq 0$, alors $h\left(\phi^{n}(P)\right) \geq h\left(x_{r}+n F_{r}\right) \geq \log |n|-c_{1}$. Si $F_{r}=0$, alors il existe $\phi_{1}$ automorphisme triangulaire de $\mathbb{A}^{r-1}$ tel que $\phi(P)=\phi_{1}^{[1]}(P)$. Ainsi $h\left(\phi^{n}(P)\right) \geq \log |n|-c_{1}$ ou bien $P$ est un point fixe. Donc pour tout point $P$ non-périodique,

$$
h\left(\phi^{n}(P)\right) \gg \ll \log |n| .
$$

Supposons $a_{i} \notin \mu^{\infty}, x_{i} \neq 0$ et $a_{i+1}=\cdots=a_{r}=1$. Alors,

$$
\begin{aligned}
h\left(a_{i}^{n} x_{i}+F_{i}^{(n)}\left(x_{i+1}, \ldots, x_{r}\right)\right) & \geq h\left(a_{i}^{n} x_{i}\right)-h\left(F_{i}^{(n)}\left(x_{i+1}, \ldots, x_{r}\right)\right)-c \\
& \geq|n| h\left(a_{i}\right)-c \log |n|-c^{\prime} \\
& \gg|n| .
\end{aligned}
$$

Donc $h\left(\phi^{n}(P)\right) \gg|n|$. De plus, pour les applications triangulaires, on a toujours $h\left(\phi^{n}(P)\right) \ll|n|$. D'où le résultat.

BULLETIN DE LA SOCiÉtÉ MATHÉMATIQUE DE FRANCE 


\section{Automorphismes du plan affine}

5.1. Preuve du corollaire B. - La classification des automorphismes du plan affine (théorème 2.15), le théorème sur les automorphismes réguliers (théorème $\mathrm{A}$ ) et le théorème sur les applications triangulaires (théorème $\mathrm{C}$ ) nous permettent essentiellement de conclure dans le cas des automorphismes du plan affine. Il faut cependant préciser qu'un produit fini d'applications de Hénon généralisées est un automorphisme régulier (lemme 2.18), que la classification sur $\mathbb{C}$ est également valable sur $k$ (remarque 2.17), que les propriétés que nous étudions sont essentiellement stables par conjugaisons (proposition 2.25), que les automorphismes réguliers ont même degré algébrique et dynamique (§2.6.1) et que les applications triangulaires sont de degré dynamique 1 (§2.2)

Nous retrouvons au passage le résultat de L. Denis sur le caractère fini de l'ensemble des points périodiques isolés d'un automorphisme du plan affine défini sur $k$. Dans sa démonstration de ce résultat L. Denis utilise les applications élémentaires normalisées.

5.2. Compléments. - Nous obtenons les résultats suivants pour les applications élémentaires normalisées.

Notation. - Nous noterons $\mu^{\infty}$ l'ensemble des racines de l'unité.

Proposition 5.1. - Soit $\phi$ un automorphisme élémentaire normalisé du plan affine, la taille des orbites des points non-périodiques est :

- Sous l'action de $\phi_{a}$,

$$
\begin{aligned}
& \triangleright \text { soit } \alpha, \beta \notin \mu^{\infty} \text { : } \\
& \quad \text { - pour } x y \neq 0 \text {, on a } N(B) \sim B\left(1 / h(\alpha, \beta)+1 / h\left(\alpha^{-1}, \beta^{-1}\right)\right) \text {; } \\
& \quad \text { - pour } y=0 \text {, on a } N(B) \sim 2 B / h(\alpha) ; \\
& \quad \text { - pour } x=0 \text {, on a } N(B) \sim 2 B / h(\beta) ; \\
& \triangleright \text { soit } \alpha \notin \mu^{\infty}, \beta \in \mu^{\infty} \text {, alors pour } x \neq 0 \text {, on a } N(B) \sim 2 B / h(\alpha) ; \\
& \triangleright \text { soit } \alpha \in \mu^{\infty}, \beta \notin \mu^{\infty} \text {, alors pour } y \neq 0 \text {, on a } N(B) \sim 2 B / h(\beta) \text {. }
\end{aligned}
$$

- Sous l'action de $\phi_{b}$ :

$\triangleright$ soit $\alpha \notin \mu^{\infty}$ et $x \neq 0$, alors on a $N(B) \sim 2 B / h(\alpha)$;

$\triangleright$ sinon, on a $N(B) \sim c(x, y) \mathrm{e}^{B}$.

- Sous l'action de $\phi_{b^{\prime}}$ :

$\triangleright$ soit $\beta \notin \mu^{\infty}$ et $y \neq 0$, alors on a $N(B) \sim 2 B / h(\beta)$;

$\triangleright$ sinon, on a $N(B) \sim c(x, y) \mathrm{e}^{B}$.

- Sous l'action de $\phi_{c}$ :

$\triangleright$ soit $\beta \in \mu^{\infty}$, on a $N(B) \sim c(x, y) \mathrm{e}^{B}$;

$\triangleright$ soit $\beta \notin \mu^{\infty}$, on a $N(B) \sim 2 B / d h(\beta)$.

- Sous l'action de $\phi_{d}$, on a $N(B) \sim c(x, y) \mathrm{e}^{B}$.

Démonstration de la proposition 5.1. - Pour chacune de ces applications, il s'agit, dans un premier temps, de déterminer l'expression des itérés de

TOME $131-2003-\mathrm{N}^{\mathrm{O}} 2$ 
celle-ci puis de déterminer la taille des orbites. Nous traitons deux cas typiques et laissons le reste des calculs au lecteur.

Ainsi, pour l'application $\phi_{a}$ nous avons : $\phi_{a}{ }^{n}(x, y)=\left(\alpha^{n} x, \beta^{n} y\right)$ pour tout $n \in \mathbb{Z}$, nous pouvons supposer que $\alpha$ et $\beta$ ne sont pas simultanément des racines de l'unité, car dans ce cas tous les points sont périodiques. Nous considèrerons le cas où $x y \neq 0$. Nous obtenons alors, pour tout $n \in \mathbb{Z}$,

$$
h\left(\alpha^{n}, \beta^{n}\right)-h\left(x^{-1}, y^{-1}\right) \leq h\left(\phi_{a}{ }^{n}(x, y)\right) \leq h\left(\alpha^{n}, \beta^{n}\right)+h(x, y) .
$$

De plus, on a $\alpha \beta \neq 0$ par hypothèse. De là,

- pour tout $n>0$,

$$
n h(\alpha, \beta)-h\left(x^{-1}, y^{-1}\right) \leq h\left(\phi_{a}^{n}(x, y)\right) \leq n h(\alpha, \beta)+h(x, y) ;
$$

- pour tout $n<0$,

$|n| h\left(\alpha^{-1}, \beta^{-1}\right)-h\left(x^{-1}, y^{-1}\right) \leq h\left(\phi_{a}^{n}(x, y)\right) \leq|n| h\left(\alpha^{-1}, \beta^{-1}\right)+h(x, y)$.

Par conséquent, $N(B) \sim B\left(1 / h(\alpha, \beta)+1 / h\left(\alpha^{-1}, \beta^{-1}\right)\right)$.

Nous considérons maintenant, le cas de $\phi_{b}$ avec $\alpha \in \mu^{\infty}$. Nous avons $\phi_{b}^{n}(P)=\left(\alpha^{n} x, y+n, 1\right)$. Nous effectuons le raisonnement sur $\mathbb{Q}$, celui-ci s'adaptant à $k$. La lettre $p$ désigne un nombre premier. Considérons $n \gg 0$ :

$$
\begin{aligned}
h\left(\phi_{b}^{n}(P)\right) & =h\left(\alpha^{n} x, y+n, 1\right) \\
& =\sum_{p} \log \max \left(|y+n|_{p}, 1\right)+\log \max \left(\left|\alpha^{n} x\right| \cdot|y+n|_{p}, 1\right) \\
& =\log n+c(y)+O\left(\frac{1}{n}\right) .
\end{aligned}
$$

En effet, si $y$ est un $p$-entier alors, $|y+n|_{p} \leq 1$ et si $y$ n'est pas un $p$-entier alors $|y+n|_{p}=|y|_{p}$. On en déduit aisément la taille des orbites.

\section{Différents exemples en dimension au moins 3}

Pour illustrer la complexité de la situation en dimension au moins 3, nous donnons différents exemples. Nous étudions tout d'abord l'application d'Anick dont les orbites sont contenues dans des quadriques. Puis, nous donnons un exemple pour lequel nous savons déterminer la taille des orbites des points non-périodiques mais pour lequel nous ne sommes pas en mesure de conclure pour les points périodiques. Il s'agit de l'application de Nagata «tordue ». Nous donnerons ensuite un moyen de construire des automorphismes affines dont la dynamique arithmétique est assez variée, il s'agit de la situation produit.

Ces différents exemples sont essentiellement tirés de travaux sur les automorphismes de l'espace affine (voir par exemple [3]). Pour une vue d 'ensemble des problèmes concernant les automorphismes de l'espace affine, nous renvoyons le lecteur au séminaire Bourbaki de H. Kraft [8]. 
6.1. Application d'Anick. — L'application d'Anick a pour expression :

$$
\sigma: \mathbb{A}^{4} \longrightarrow \mathbb{A}^{4}, \quad(x, y, z, w) \longmapsto(x-(x z+y w) w, y+(x z+y w) z, z, w) .
$$

Cette application qui est un contre-exemple potentiel à la conjecture des polynômes modérés (Tame generator conjecture), est construite comme l'exponentielle d'une dérivation localement nilpotente; en effet, si $D=z \delta_{y}-w \delta_{z}$ et si l'on choisit la constante $a=x z+y w$, alors $\sigma=\exp (a D)$. Le degré dynamique de l'application d'Anick est égal à 1 .

Nous pouvons calculer directement les itérés de cette application, nous obtenons alors le résultat suivant :

Proposition 6.1. - L'application d'Anick $\phi$ vérifie les assertions suivantes :

- L'application $\phi$ n'admet pour points périodiques que des points fixes, ils sont situés sur la quadrique d'équation $x z+y w=0$.

- Les orbites des points non-périodiques de $\phi$ sont contenus dans les quadriques d'équations $x z+y w=c$, où $c$ est une constante non nulle, elles ont pour taille $N(B) \sim c(x, y, z, w) \mathrm{e}^{B}$.

Démonstration. - Les itérés de $\phi$ s'expriment sous la forme

$$
\phi^{n}(x, y, z, w)=(x-n(x z+y w) w, y+n(x z+y w) z, z, w)
$$

pour tout $n \in \mathbb{Z}$. De plus les coordonnées de $\phi^{n}$ notées $x_{n}, y_{n}, z_{n}, w_{n}$ vérifient pour tout $n x_{n} z_{n}+y_{n} w_{n}=x y+z w$ avec $(x, y, z, w)=\left(x_{0}, y_{0}, z_{0}, w_{0}\right)$. Les orbites des points non-périodiques sont donc contenues dans des quadriques. À partir de l'expression des itérés et à l'aide de la définition de la hauteur d'un point nous déterminons la taille des orbites des points non-périodiques. La preuve est identique à celle effectuée pour les applications élémentaires normalisées.

6.2. Application de Nagata «tordue». — L'application de Nagata « tordue » est ainsi dénommée car c'est la composée de l'application de Nagata (qui est un contre-exemple potentiel à la conjecture des polynômes modérés [12]) avec l'application $\tau(X, Y)=(Y, X)$. Cette application est une illustration de la variété de la situation en dimension au moins 3 . Nous pouvons obtenir des résultats sur la taille des orbites de ses points non-périodiques.

Nous avons «tordu » l'application de Nagata car nous sommes ramenée à l'étude d'une famille paramétrée d'automorphismes réguliers du plan affine. Nous obtenons le résultat suivant :

ThÉORÈme 6.2. - L'application de Nagata "tordue» définie de la manière suivante :

$$
\phi: \mathbb{A}^{3} \longrightarrow \mathbb{A}^{3}, \quad(X, Y, Z) \longmapsto\left\{\begin{array}{l}
Y-2\left(Y Z+X^{2}\right) X-\left(Y Z+X^{2}\right)^{2} Z, \\
X+\left(Y Z+X^{2}\right) Z, \\
Z
\end{array}\right.
$$

TOME $131-2003-\mathrm{N}^{\mathrm{O}} 2$ 
possède les propriétés suivantes.

Soit $P$ un point non-périodique, alors :

1) Soit $P$ de coordonnées $(X, Y, Z)$ avec $Z \neq 0$;

$\triangleright \log \left(h\left(\phi^{n} P\right)\right) \sim|n| \log 4$ quand $|n| \rightarrow \infty$,

$\triangleright N(\phi, P, B) \sim 2 \log B / \log 4$.

2) Soit $P$ de coordonnées $(X, Y, 0)$; alors

$\triangleright \log \left(h\left(\phi^{n} P\right)\right) \sim|n| \log 3$ quand $|n| \rightarrow \infty$

$\triangleright N(\phi, P, B) \sim 2 \log B / \log 3$.

Démonstration. - Nous nous ramenons à une famille d'applications paramétrées par $m$. Pour $m \neq 0$, nous sommes ramenée à l'étude de

$\phi(m)(X, Y)=\left(Y-2 m X Y-2 X^{3}-m\left(m^{2} Y^{2}+2 m X^{2} Y+X^{4}\right), X+m^{2} Y+m X^{2}\right)$

et pour $m=0$ à celle de l'application

$$
\phi(0)(X, Y)=\left(Y-2 X^{3}, X\right) .
$$

Ces deux applications sont des automorphismes réguliers, d'où le résultat à l'aide du théorème $\mathrm{A}$.

Nous avons $Z(\phi) \cap Z\left(\phi^{-1}\right)=\{P=(X, Y, 0,0)\}$ et l'application de Nagata «tordue » est une application de degré dynamique égal à 5 .

Remarque 6.3. - Dans le cas de l'application de Nagata «tordue », nous ne pouvons répondre qu'à la seconde question. En effet, nous obtenons ce résultat à l'aide d'une famille paramétrée d'automorphismes, par conséquent nous obtenons une majoration de la hauteur des points périodiques qui dépend du paramètre, nous ne pouvons donc conclure. Nous pouvons simplement dire que dans chaque plan d'équation $z=\mathrm{C}^{\text {te }}$, l'ensemble des points périoques est un ensemble de hauteur bornée, il est a fortiori fini sur $k$

Il est possible de généraliser cette méthode sous certaines conditions.

Nous montrons maintenant une méthode qui permet de construire des applications dont le comportement du point de vue de leur dynamique arithmétique est extrêmement varié.

6.3. Situation produit. - Si $\phi_{i} \in \operatorname{Aut}\left(\mathbb{A}^{r_{i}}\right)$, pour $1 \leq s$ nous définisons l'application $\Phi \in \operatorname{Aut}\left(\mathbb{A}^{r_{1}+\cdots+r_{s}}\right)$ de la façon suivante :

$$
\Phi\left(x_{1}, \ldots, x_{r_{1}+\cdots+r_{s}}\right)=\left(\phi_{1}\left(x_{1,1}, \ldots, x_{r_{1}, 1}\right), \ldots, \phi_{s}\left(x_{1, s}, \ldots, x_{r_{s}, s}\right)\right) .
$$

La connaissance du comportement des orbites de chaque application $\phi_{i}$, nous donne le comportement des orbites de l'automorphisme $\phi$. 
Nous pouvons par exemple considérer l'exemple suivant : soit $\phi_{1} \in \operatorname{Aut}\left(\mathbb{A}^{2}\right)$ une application triangulaire. Soit $\phi_{2}$ une application de Hénon généralisée. L'automorphisme $\Phi$ a pour expression

$$
\Phi=(\alpha x+q(y), \beta y+\gamma, p(z)-a t, z) .
$$

De plus, nous avons $\delta(\Phi)=\delta\left(\phi_{2}\right)$.

Dynamique arithmétique de $\Phi$

- L'automorphisme $\Phi$ admet un nombre fini de points périodiques dans $k$.

- Soit $P=(x, y, z, t)$; si $P_{1}=(x, y) \notin \operatorname{Per}\left(\phi_{1}, k\right)$ et $P_{2}=(z, t) \in \operatorname{Per}\left(\phi_{2}, k\right)$, alors

$$
N(\Phi, P, B)=N\left(\phi_{1}, P_{1}, B\right) .
$$

Suivant le type d'automorphisme triangulaire et le point considéré, différents cas se présentent. Les tailles des orbites sont de la forme $N(B) \gg \ll B$ ou $\log N(B) \gg \ll B$.

- Soit $P=(x, y, z, t)$; si $P_{1}=(x, y)$ et $P_{2}=(z, t) \notin \operatorname{Per}\left(\phi_{2}, k\right)$, alors

$$
N(\Phi, P, B) \sim 2 \frac{\log B}{\log \delta(\Phi)} .
$$

En effet, $\phi_{2}$ est une application de Hénon généralisée d'où la première taille d'orbite, quant à $\phi_{1}$ c'est une application triangulaire.

Pour le troisième cas, il s'agit de regarder la croissance des hauteurs des itérés. Ainsi $\phi_{2}$ est une application de Hénon généralisée donc les hauteurs des itérés sont de type exponentiel et les hauteurs des itérés de $\phi_{2}$ sont de type polynomial. D'où le résultat sachant que $\delta(\Phi)=\delta\left(\phi_{2}\right)$.

REMARQUe 6.4. - Nous avons construit une application dont l'intersection de $Z(\Phi)$ avec $Z\left(\Phi^{-1}\right)$ est réduite à un point et qui possède des orbites de différentes tailles. Ainsi, l'hypothèse de régularité qui nous permet de conclure à des orbites de la forme $N(B) \sim c \log _{d} B$ est bien nécessaire.

\section{BIBLIOGRAPHIE}

[1] Bass (H.), Connell (E.H.) \& Wright (D.) - The Jacobian conjecture : reduction of degree and formal expansion of the inverse, Bull. Amer. Math. Soc. (N.S.), t. 7 (1982), pp. 287-330.

[2] Denis (L.) - Points périodiques des automorphismes affines, J. reine angew. Math., t. 467 (1995), pp. 157-167.

[3] VAn Den Essen (A.), éd. - Automorphisms of affine spaces, Dordrecht, Kluwer Academic Publishers, 1995.

[4] Fornaess (J.E.) \& Sibony (N.) - Complex dynamics in higher dimensions, in Complex potential theory (Montreal, PQ, 1993) (Dordrecht),

TOME $131-2003-\mathrm{N}^{\mathrm{O}} 2$ 
NATO Adv. Sci. Inst. Ser. C Math. Phys. Sci., vol. 439, Kluwer Acad. Publ., 1994, pp. 131-186.

[5] Friedland (S.) \& Milnor (J.) - Dynamical properties of plane polynomial automorphisms, Ergodic Theory Dynamical Systems, t. 9 (1989), pp. 67-99.

[6] Hindry (M.) \& Silverman (J.) - Diophantine geometry, an introduction, Springer Verlag, 2000.

[7] Jung (H.W.E.) - Über ganze birationale Transformationen der Ebene, J. reine angew. Math., t. 184 (1942), pp. 161-174.

[8] Kraft (H.) - Challenging problems on affine n-space, in Séminaire Bourbaki 1994/95, Astérisque, vol. 237, Société Mathématique de France, 1996, exp. 802, pp. 295-317.

[9] VAN DER KULK (W.) - On polynomial rings in two variables, Nieuw Arch. Wiskunde (3), t. 1 (1953), pp. 33-41.

[10] Marcello (S.) - Sur la dynamique arithmétique des automorphismes affines, Thèse, Université Paris 7 (Denis Diderot), 2000.

[11] Sur les propriétés arithmétiques des itérés d'automorphismes réguliers, C. R. Acad. Sci. Paris Sér. I Math., t. 331 (2000), pp. 11-16.

[12] Nagata (M.) - On automorphism group of $k[x, y]$, Lectures in Mathematics, vol. 5, Department of Mathematics, Kyoto University, Kinokuniya Book-Store Co., Ltd., Tokyo, 1972.

[13] Northcott (D.G.) - Periodic points on an algebraic variety, Ann. of Math., t. 51 (1950), pp. 167-177.

[14] Peyre (E.) - Points de hauteur bornée et géométrie des variétés d'après Y. Manin et al., in Séminaire Bourbaki 2000/2001, Astérisque, vol. 282, Société Mathématique de France, 2002, exp. 891, pp. 323-344.

[15] Sibony (N.) - Dynamique des applications rationnelles de $\mathbb{P}^{k}$, in Dynamique et géométrie complexes, Panoramas et Synthèses, vol. 8, Société Mathématique de France, 1999, pp. 97-195.

[16] Silverman (J.) - Geometric and arithmetic properties of the Hénon map, Math. Z., t. 215 (1994), pp. 237-250.

[17] Yagzhev (A.V.) - On Keller's problem, Siberian Math. J., t. 21 (1980), pp. $747-754$. 